\title{
Primary Hydatid Cyst of the Diaphragm: A Case Report
}

Mihai Dumitrescu, Ciprian Bolca*a and loan Cordoș

Department of Thoracic Surgery, "Marius Nasta" Institute of Pneumology, Bucharest, Romania

\begin{abstract}
Hydatid disease is a parasitic disease endemic in Romania which occurs frequently in liver and lungs, but it can also be present in almost any part of the body. We present the rare case of a 37-year-old male which was admitted in our service with a large hydatid cyst at the level of the right thoracic outlet and we were not able initially to establish its exact origin, liver or right lung. After imagistic investigations we decided for an abdominal approach considering that the cyst was liver related. Intraoperative findings showed a primary hydatid cyst of the diaphragm, a very rare entity that was successfully treated by the mentioned approach.
\end{abstract}

Keywords Echinococcus Granulosus; Hydatid Cyst; Diaphragm

\section{Introduction}

Hydatid disease (HD) is a parasitic disease caused by Echinococcus granulosus. The disease is endemic in Eastern Europe, including Romania which has a morbidity index of 5.6 to 100.000 people [1]. It occurs frequently in the liver (59-75\%), but it can also be present in the lung (27\%), kidney (3\%), bone (1-4\%) and diaphragm (1\%), the last being generally associated with liver hydatidosis $[2,3]$. In this report we present a case with primary hydatid cyst of the diaphragm, with no other cysts present, which was successfully managed in our department.

\section{Case Report}

A 37-year-old male, with no history of surgery, was admitted in our department for thoracoabdominal pain for 3 days. At admission the patient was without fever and in good general condition, despite suffering of thoracoabdominal pain. Laboratory tests showed slightly elevated leucocytes and no eosinophilia was present. Chest X-ray performed at admittance revealed right-sided subpulmonic opacity with elevation of the right hemidiaphragm. The CT scan performed the next day showed a giant hydatid cyst extending from the abdominal cavity in to the right thoracic cavity but did not offer any information regarding the origin of the hydatid cyst (Figure 1). As it was considered that we deal with a liver cyst, we have decided for an abdominal approach.

An exploratory laparotomy was performed on the third day, during which time we discovered a giant hydatid cyst (Figure. 2) between the muscle fibers of the diaphragm, without liver or lung involvement, pushing down on the liver and compressing the inferior right pulmonary lobe. Careful dissection of the muscle fibers was carried out in order to avoid spillage in the abdominal cavity (Figure 3). Upon reaching the membrane of the cyst we decided to first evacuate the content with the help of a $16 \mathrm{G}$ needle. Next we irrigated the site with $90 \%$ alcohol solution in order to inactivate the remaining content, and finally we evacuated the cyst again using the same $16 \mathrm{G}$ needle.

Removal of the cyst's membrane went without incidents, and closure of the defect was performed with individual silk suture points. Histological examination established the presence of muscle fibers in the pericyst and confirmed the diagnosis of hydatid cyst.

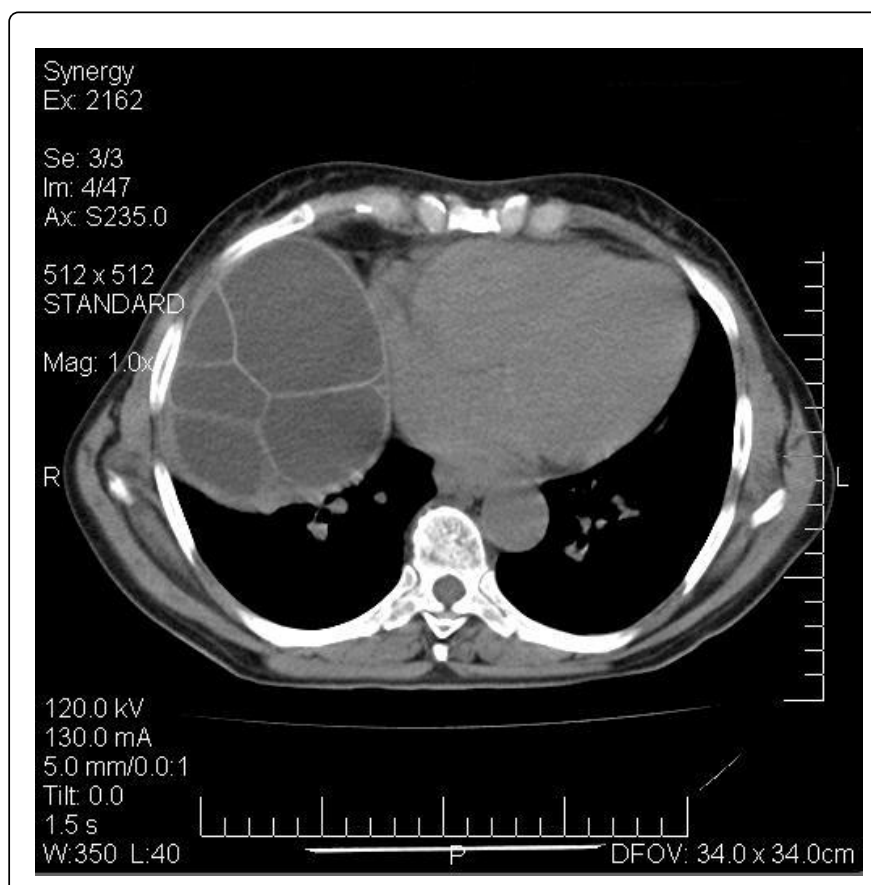

Figure 1: Diaphragmatic Hydatid Cyst - CT

Postoperative recovery was uneventful and the patient was discharged on the fourth postoperative day and directed to a parasitological department for medical treatment.

*Corresponding author: Ciprian Bolca, Department of Thoracic Surgery, "Marius Nasta" Institute of Pneumology, Șoseaua Viilor No 90, 050152, București,

Romania, Tel: +40 (0) 213356910-14/1517; E-mail: bolcaciprian@gmail.com

Received: February 24, 2014, Accepted: March 31, 2014, Published: April 5, 2014

Citation: Citation: Dumitrescu M, Bolca C, Cordos I. Primary Hydatid Cyst of the Diaphragm: A Case Report. Journal of Surgery [Jurnalul de chirurgie] 2014; 10(3): 253-254. doi:10.7438/1584-9341-10-3-13

Copyright: @ 2014 Dumitrescu M, et al. This is an open-access article distributed under the terms of the Creative Commons Attribution License, which permits unrestricted use, distribution, and reproduction in any medium, provided the original author and source are credited. 


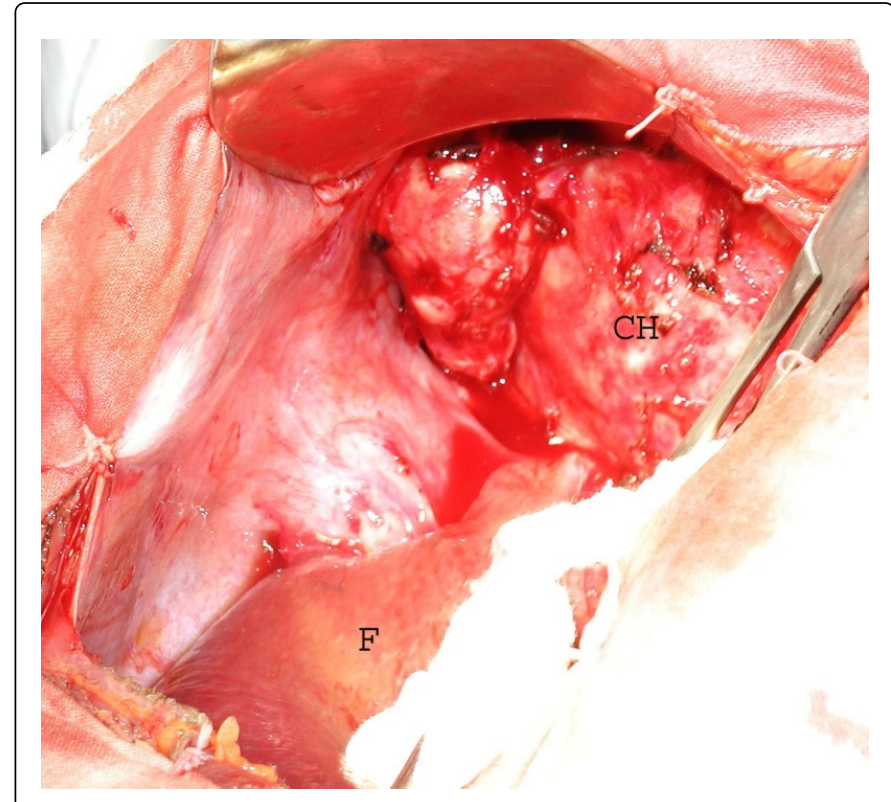

Figure 2: Intraoperative Aspect

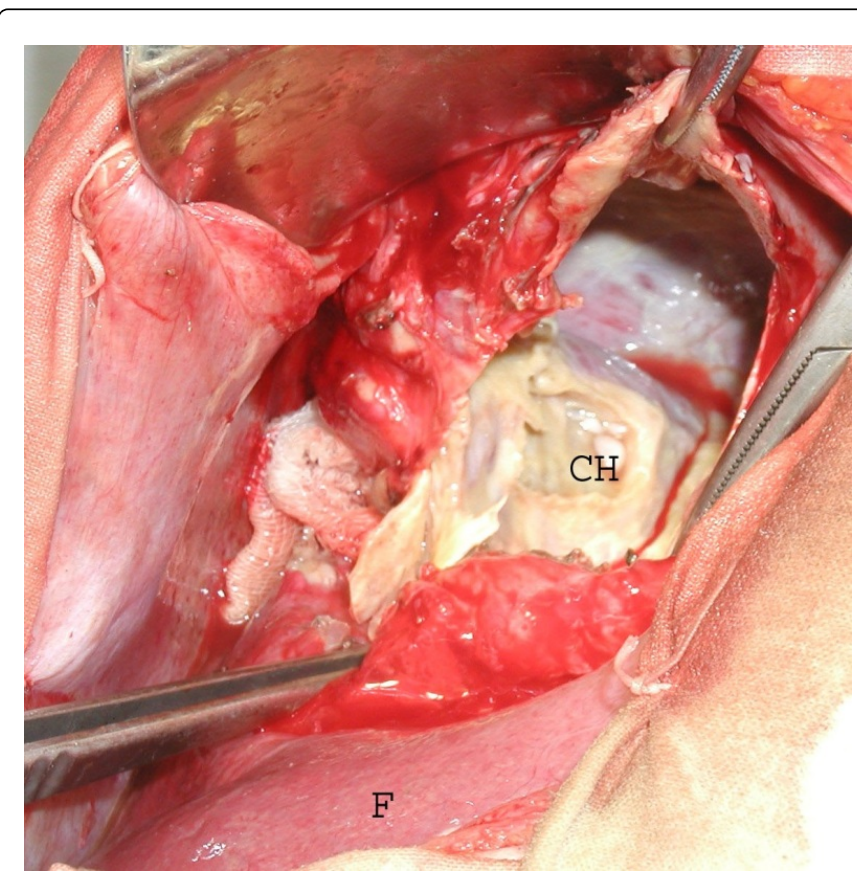

Figure 3: Diaphragmatic Hydatid Cyst is Opened (Intraoperative Aspect)

\section{Discussion}

The primary hydatid cyst of the diaphragmatic is a rare clinical entity defined as a cyst with no involvement of the pulmonary parenchyma and with no transmission from the abdomen to the thorax, whereas a secondary cyst is most likely caused by transdiaphragmatic migration from the posterior segments of the right hepatic lobe $[4,5]$. Diaphragmatic localization of the hydatid cyst is most likely caused by dissemination of the embryos through the arterial circulation [6]. Diagnosis is confirmed by the presence of muscle fibers in the pericyst during histological examination [7]. In the preoperative period careful topographic diagnosis between the diaphragm, lung, liver and abdominal localizations should be made either by use of CT or MRI examinations [8]. Although total excision of the cyst through the thoracotomy is considered an excellent approach [9], we prefer using the laparotomy in cases in which the CT examination doesn't rule out liver involvement. Also, we have achieved good long term results without excising completely the pericyst, simple removal of the membrane being sufficient. Suturing of the diaphragmatic defect is a must in order to avoid possible herniation and to ensure good pulmonary function.

\section{Conclusion}

The primary hydatid cyst of the diaphragmatic is a rare clinical entity and the diagnosis is challenging. Although total excision of the cyst through the thoracotomy is considered an excellent approach, we prefer using the laparotomy in cases in which the CT examination doesn't rule out abdominal viscera involvement. A good diaphragmatic suture is mandatory to avoid possible herniation and respiratory dysfunction.

\section{Conflict of interest}

Authors have no conflict of interests to disclose.

\section{References}

1. Barabas E (2007) Epidemiology of hydatid disease caused by E. granulosus and E. multilocularis in Romania. Rev Rom de Parazitologie 17: $165-168$

2. Yuksel M, Demirpolat G, Sever A, Bakaris S, Bulbuloglu E, et al. (2007) Hydatid disease involving some rare locations in the body: a pictorial essay. Korean J Radiol 8: 531-540.

3. Ersoy G, Yildirir C, Gökgöz S, Ozer T, Tulpar A (1993) Hydatid cyst of diaphragm--a case of hydatid cyst with rare localisation. Mater Med Pol 25: $109-112$.

4. Gursoy S, Ucvet A, Tozum H, Erbaycu AE, Kul C, et al. (2009) Primary intrathoracic extrapulmonary hydatid cysts: analysis of 14 patients with a rare clinical entity. Tex Heart Inst J 36: 230-233.

5. Polat P, Kantarci M, Alper F, Suma S, Koruyucu MB, et al. (2003) Hydatid disease from head to toe. Radiographics 23: 475-494.

6. De Vega DS, Vazquez E, Calvo E, Tamames S, Tamames S (1991) [Hydatid cyst of the diaphragm. Apropos of a case]. J Chir (Paris) 128: 76-78.

7. Eren S, Ulku R, Tanrikulu AC, Eren MN (2004) Primary giant hydatid cyst of the diaphragm. Ann Thorac Cardiovasc Surg 10: 118-119.

8. OÄŸuzkaya F, AkÃ§ali Y, Kahraman C, EmiroÄŸullari N, Bilgin M, et al. (1997) Unusually located hydatid cysts: intrathoracic but extrapulmonary. Ann Thorac Surg 64: 334-337.

9. Ulkü R, Eren N, Cakir O, Balci A, Onat S (2004) Extrapulmonary intrathoracic hydatid cysts. Can J Surg 47: 95-98. 